\title{
Intrinsic current-voltage properties of nanowires with four-probe scanning tunneling microscopy: A conductance transition of $\mathrm{ZnO}$ nanowire
}

\author{
X. Lin, X. B. He, T. Z. Yang, W. Guo, D. X. Shi, and H.-J. Gao a) \\ Beijing National Laboratory for Condensed Matter Physics, Institute of Physics, Chinese Academy of \\ Sciences, P.O. Box 603, Beijing 100080, China \\ D. D. D. Ma and S. T. Lee \\ Center of Super-Diamond and Advanced Films (COSDAF) and Department of Physics and Materials \\ Science, City University of Hong Kong, Hong Kong SAR, China \\ F. Liu \\ Department of Materials Science and Engineering, University of Utah, Salt Lake City, Utah 84112
}

X. C. Xie

Department of Physics, Oklahoma State University, Stillwater, Oklahoma 74078

(Received 13 April 2006; accepted 30 May 2006; published online 24 July 2006)

\begin{abstract}
We report intrinsic current-voltage properties of $\mathrm{ZnO}$ nanowire measured by a four-tip scanning tunneling microscopy (F-STM). It is found that after bending the nanowire with the F-STM the conductance is reduced by about five orders of magnitude. The cathodoluminescent spectra indicate that the $\mathrm{ZnO}$ nanowires contain a sizable amount of defects in the surface region, responsible for their conduction. It is suggested that the observed huge conductance changes are caused by the shifting of the surface defect states in the $\mathrm{ZnO}$ nanowires in response to the applied surface strain.

(C) 2006 American Institute of Physics. [DOI: 10.1063/1.2234293]
\end{abstract}

The electronic structures and transport properties of solid materials depend strongly on strain. Such dependence has provided a unique and useful mechanism in designing electronic devices, such as in the conventional strain engineering of band structures of semiconductor superlattices. The electronic structures and transport properties of solidstate nanostructures are expected to be even more sensitive to strain due to their reduced size and dimensionality. ${ }^{1-3}$

The "smallness" of nanostructures allows them to sustain a much larger strain than their bulk counterparts, while maintaining structural integrity (e.g., coherent without dislocation formation ${ }^{4}$ ). This provides a high degree of flexibility in designing nanoelectromechanical devices and sensors, opening up a new emerging field of flexible electronics. ${ }^{5-8}$ There have been increased recent interests in understanding the transport properties and their strain dependence ${ }^{1,9,10}$ of nanostructures and nanoscale systems. The work to date, however, has mostly focused on how strain changes the intrinsic electronic structures and transport properties of nanostructures, such as the modification of host electron band structure under strain. Here, using $\mathrm{ZnO}$ nanowires as a model system, we demonstrate that strain can also have a profound effect on the defect electronic structure of a nanostructure and, hence, change its transport property.

We have developed a four-tip scanning tunneling microscope (F-STM) setup, ${ }^{11,12}$ which consists of four specially designed STMs, capable of performing the dual functions to bend the wire and to measure the conductance and the current-voltage $(I-V)$ curves in situ on the same sample, here a single $\mathrm{ZnO}$ nanowire. We found that the electrical conductance of the $\mathrm{ZnO}$ nanowires changes by five orders of magnitude when they are bent by STM tips. Both the bending process and the conductance transition are completely re-

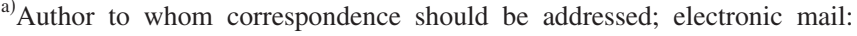
hjgao@aphy.iphy.ac.cn
}

versible. Cathodoluminescent (CL) spectra and temperature dependence of the conductivity indicate that our $\mathrm{ZnO}$ nanowire samples contain a sizable amount of defects, making the original wire conducting. Consequently, we deduce that the huge conductance change is caused by the shifting of defect electronic states induced by the external strain.

The ZnO-nanowire samples used in our experiments were synthesized via reduction and oxidation of $\mathrm{ZnS}$ powder $^{13}$ and dispersed on a silicon wafer coated with a 500-nm-thick silicon dioxide layer as insulator. The STM tips were made from a tungsten or gold wire using the standard preparation process. All measurements are performed in dark environment with the electron beam of scanning electron microscopy shielded.

First, before performing the bending experiments, we characterized our $\mathrm{ZnO}$-nanowire samples, by measuring the "intrinsic" $I-V$ characteristics of a single $\mathrm{ZnO}$ nanowire using the four-terminal method in our F-STM. Figure 1 shows one typical $I-V$ curve of the $\mathrm{ZnO}$ nanowire, displaying a linear relationship with a resistance of $\sim 25.9 \mathrm{k} \Omega$. The relatively high metal-like conductivity $(4.1 \mathrm{~S} / \mathrm{cm}$ in this case) indicates that the $\mathrm{ZnO}$ nanowires used here are distinctively

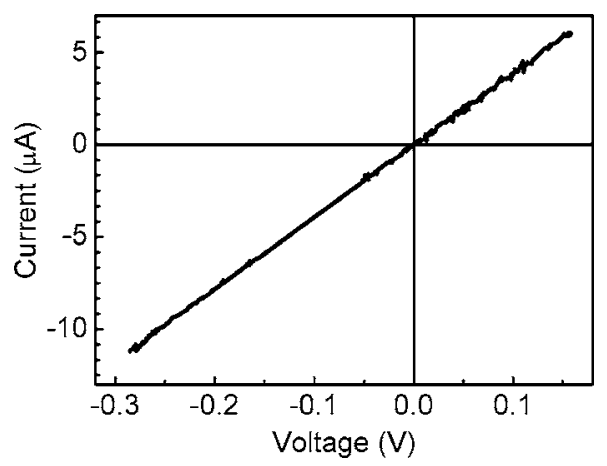

FIG. 1. I-V curve obtained by the four-terminal method. 


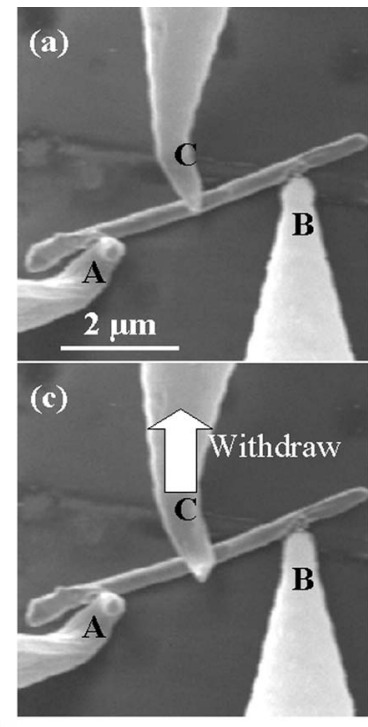

(e)
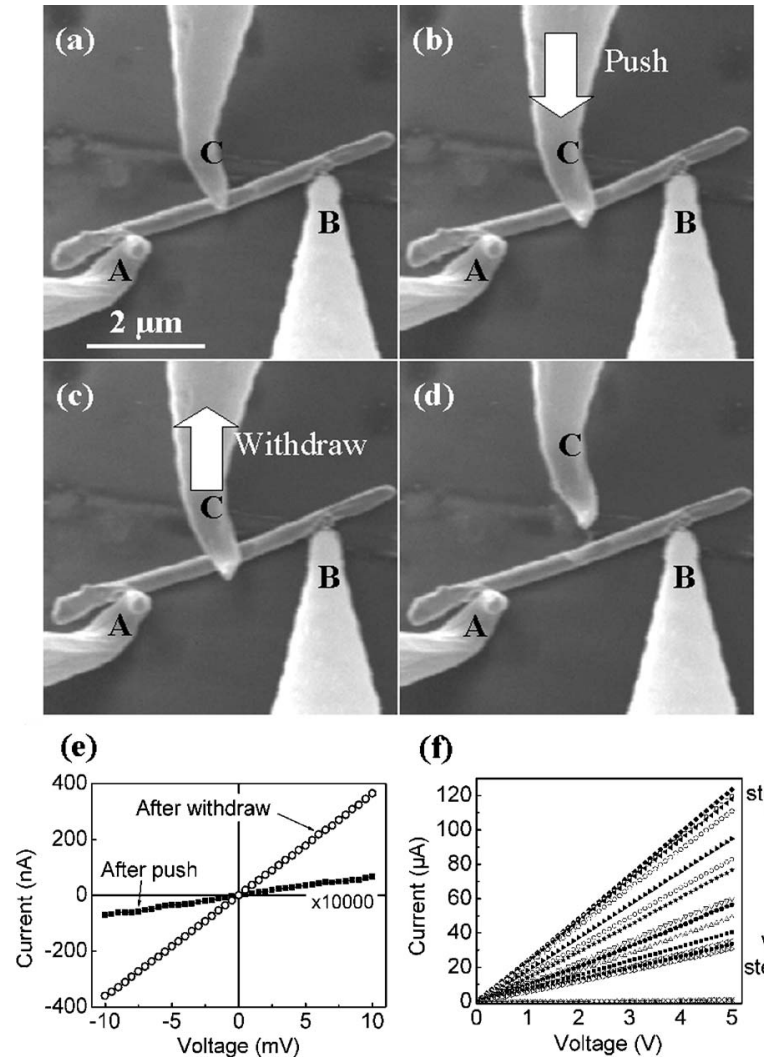

(f)

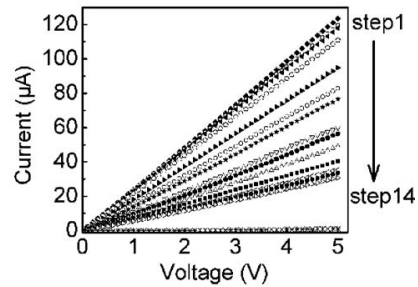

FIG. 2. (a) Two outer tips fixed the nanowire and measure the $I-V$ curves; (b) the middle probe pushed down and bent the nanowire; (c) the middle tip retracted and loosened the nanowire; (d) the middle tip moved away; (e) curve ( $\square$ ) corresponds to (b) and curve (O) corresponds to (c); (f) another experiment: pushing the nanowire with several small steps, and the corresponding $I-V$ curves after each step.

different from those conventional undoped $\mathrm{ZnO} .{ }^{14}$ Besides the four-terminal method, two-terminal method was used to take a series of data with different tip separations for calculating the contact resistance and make sure if there is a nonOhmic contact. In our experiment, no notable contact resistance was observed and the linear characteristic of the $I-V$ curve up to $\pm 5 \mathrm{~V}$ shows that the contact is Ohmic without Schottky barrier.

Next, we measured the $I-V$ curves of the $\mathrm{ZnO}$ nanowires while they are bent using the F-STM. Figures 2(a)-2(d) illustrate the bending process. Two tips (tips A and B) were used to support (fix) the two ends of a nanowire, and a third tip (tip C) was placed in the middle to push (bend) the nanowire. During the bending process, the electrical measurement was conducted in situ through the two outside tips.

Figure 2(e) shows, respectively, the $I-V$ curves of a $\mathrm{ZnO}$ nanowire at the maximum bent position and the final restored position after the middle tip $\mathrm{C}$ was withdrawn. The resistance of the maximally bent nanowire [Fig. 2(d)] is about $1.42 \mathrm{G} \Omega$ derived from the ( $\mathbf{\square})$ curve of Fig. 2(e), which is about five orders of magnitude lower than that of the original unbent wire $(\sim 25.9 \mathrm{k} \Omega)$. From the amount of push of the middle tip $\mathrm{C}$ and the dimension (length and diameter) of the nanowire, we estimated using the beam bending theory ${ }^{15,16}$ that the bending introduced a maximum of about $\pm 1.3 \%$ tensional strain in the surface regions of the nanowire. The resistance at the final restored nanowire position after tip $\mathrm{C}$ was withdrawn [Fig. 2(d)] is about $27.6 \mathrm{k} \Omega$ derived from the $(\bigcirc)$ curve of Fig. 2(e), which is about the same as that of the original unbent wire $(\sim 25.9 \mathrm{k} \Omega)$. This indicates that the im-

Downloaded 09 Jan 2007 to 155.98.5.35. Redistribution subject to

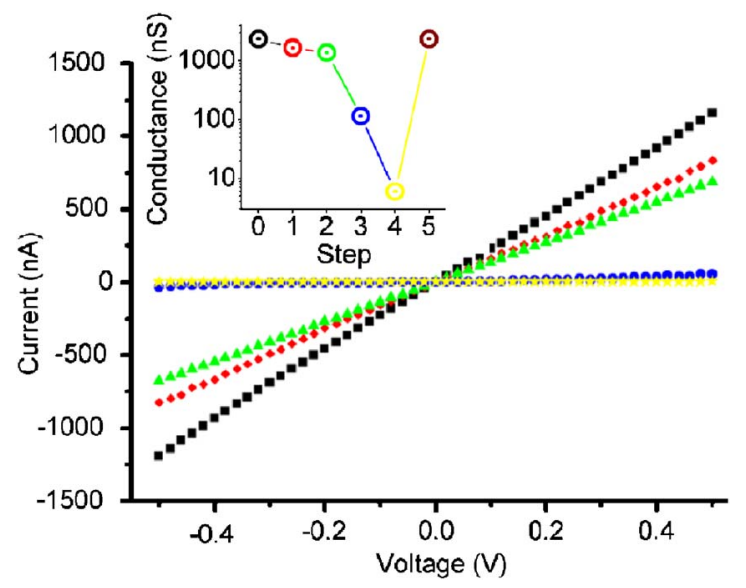

FIG. 3. (Color online) Conductance transition corresponds to the bending process at low temperature $(30 \mathrm{~K})$. Conductance dropped when bending the nanowire step-by-step (from the first step to the fourth step) and reverted to the initial state when the middle tip was retreated to release the nanowire to its original shape (fifth step).

posed bending deformation of the nanowire is essentially elastic and the induced change of conductance is reversible!

In order to reveal the detailed dependence of conductance on bending strain, we have measured $I-V$ curves as the tip $\mathrm{C}$ was pushing down in incremental steps, as shown in Fig. 2(f). The conductance, i.e., the slope of the $I-V$ curves, decreases continually as the pushing and hence the surface strain increase. We have also performed the same transport experiments at low temperature $(\sim 30 \mathrm{~K})$ and the measured $I-V$ curves are shown in Fig. 3. The results are very similar to those at room temperature, as shown in Fig. 2. In general, the conductance continued to decrease as the middle tip was pushed down and the strain in the $\mathrm{ZnO}$ nanowire increases. After the middle tip was withdrawn, the $\mathrm{ZnO}$ nanowire at the restored position after bending exhibits a conductance almost the same as that at the initial configuration before bending.

We can safely rule out the possibility that the change of $\mathrm{ZnO}$ nanowire conductance might be caused by changing contact condition during bending or influenced by tip condition. This is because the measured conductance is the same before and after the bending operation by tip movement, which is also very close to the original value obtained from the four-terminal-method measurement. Furthermore, as we had mentioned before, a series of two-terminal-method measurement and the $I-V$ curves show that the contact is Ohmic and the contact resistance is unapparent compared to the resistance of $\mathrm{ZnO}$ nanowire. Besides tungsten, we also used gold tips, which have produced similar results as tungsten tips. Therefore, we concluded the change of $\mathrm{ZnO}$ nanowire conductance must be caused by the change of electronic structure of $\mathrm{ZnO}$ induced by bending strain, as we discuss below.

In order to reveal the underlying mechanisms for such huge conductance change in $\mathrm{ZnO}$ nanowires induced by bending (strain), we analyze the CL spectra of the $\mathrm{ZnO}$ nanowires to understand the origin of its conduction at the "normal" state without bending. Figures 4(a) and 4(b) show the $\mathrm{CL}$ spectra of a single $\mathrm{ZnO}$ nanowire obtained at room temperature $(\mathrm{RT})(300 \mathrm{~K})$ and low temperature $(\sim 30 \mathrm{~K})$, respectively. The RT spectrum contains a sharp and high peak of near-UV emission at $388 \mathrm{~nm}$ and a shallow peak of green emission around $530 \mathrm{~nm}$. The near-UV emission agrees with the band gap of bulk $\mathrm{ZnO}$ (around $380 \mathrm{~nm}$ ) $^{17-19}$ coming from AIP license or copyright, see http://apl.aip.org/apl/copyright.jsp 

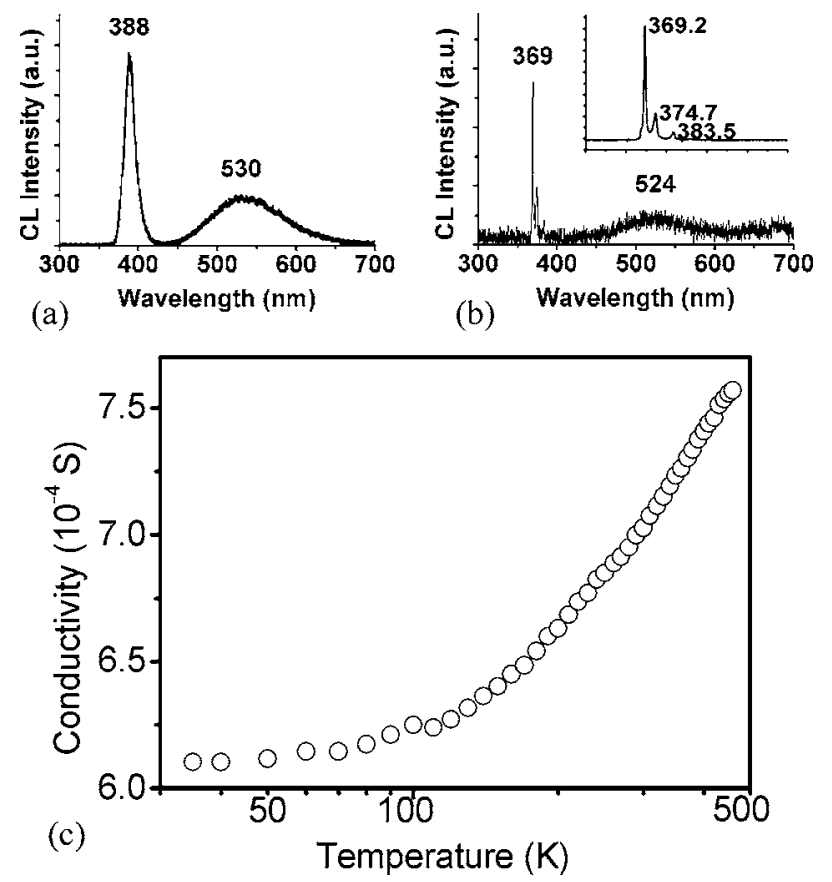

FIG. 4. CL spectra of single $\mathrm{ZnO}$ nanowire and temperature dependence of conductivity. CL spectra were excited by an electron beam of $5 \mathrm{keV}$. (a) CL spectrum recorded at room temperature. (b) CL spectrum recorded at low temperature $(30 \mathrm{~K})$. The inset shows the detail of the low-temperature spectrum from 340 to $440 \mathrm{~nm}$. (c) Temperature dependence of conductivity of a single $\mathrm{ZnO}$ nanowire. The horizontal axis is in a logarithmic scale. All experimental data were obtained with the four-terminal-method measurement.

the recombination of free excitons ${ }^{20}$ the green emission is likely due to a local energy level in the band gap. ${ }^{21,22}$ At low temperature, both emissions exhibit a blueshift to smaller wavelength. The inset of Fig. 4(b) further shows that the near-UV emission consists of three peaks at 369.2, 374.7, and $383.5 \mathrm{~nm}$, respectively.

The CL spectra indicate that there are plenty of defects in the $\mathrm{ZnO}$ nanowire [Figs. 4(a) and 4(b)], which can act as a dopant responsible for the observed high conductivity at the normal state. Previous studies have shown that it is the defects at the surface of a $\mathrm{ZnO}$ nanowire that dominate its conductivity. ${ }^{16,23,24}$ The defects at the surface produce a large number of surface states in the gap crossing the Fermi level, making our $\mathrm{ZnO}$ nanowires behave like a weakly conductive two-dimensional metal with a low resistance in the normal state (i.e., the strain-free state). The conduction electrons likely flow through the surface region of the $\mathrm{ZnO}$ nanowires.

The temperature dependence of the normal-state conductivity is also measured, as shown in Fig. 4(c). There are two key points to be observed in Fig. 4(c). First, the magnitude of conductivity is much higher than what has been reported previously for $\mathrm{ZnO}$ nanowire. ${ }^{14}$ Second, the conductance changes only by $\sim 19 \%$ over a large temperature range $(35-460 \mathrm{~K})$. These two facts suggest that doping caused the electron transport of our $\mathrm{ZnO}$ nanowires to be similar to that of noncrystalline metals.

Now, we establish that the electron transport of the $\mathrm{ZnO}$ nanowire is characterized as a weakly disordered metal. When the $\mathrm{ZnO}$ nanowire is bent, the bending induced surface strain will greatly shift the positions of the surface states changing their relative position to the Fermi level. Especially, if the strain makes the Fermi level move out of the range of surface states, then the conductance of the $\mathrm{ZnO}$ nanowire will decrease continuously as strain (bending) increases. When the strain is removed, the conductance will return to its normal state as the surface states restore their original positions overlapping with the Fermi level. Thus, we postulate that strain has a strong effect in changing the alignment of the Fermi level and the electron states of defects in $\mathrm{ZnO}$ nanowires whose surface contains a large amount of defects. This postulation remains to be confirmed by further studies, e.g., by first-principles calculations.

In conclusion, we discover a strain-induced conductance transition in $\mathrm{ZnO}$ nanowires. The mechanism of the conductance transition is attributed to the strain induced change of surface states. Our findings demonstrate a unique example of strain-induced change in defect electronic states of nanostructures, rather than change in host electronic states of nanostructures as shown extensively by previous studies. We believe the discovered phenomena and underlying mechanisms are not only of broad scientific interests but also of great technological significance, because they may occur generally in semiconductor nanostructures with a high level of intrinsic defects or extrinsic dopants.

This work was partially supported by grants from NSFC with Grant No. 90406022 and the Chinese Academy of Sciences.

${ }^{1}$ T. W. Tombler, C. W. Zhou, L. Alexseyev, J. Kong, H. J. Dai, L. Lei, C. S. Jayanthi, M. J. Tang, and S. Y. Wu, Nature (London) 405, 769 (2000).

${ }^{2}$ J. Q. Lu, J. Wu, W. H. Duan, F. Liu, B. F. Zhu, and B. L. Gu, Phys. Rev. Lett. 90, 156601 (2003)

${ }^{3}$ J. Zang, A. Treibergs, Y. Han, and F. Liu, Phys. Rev. Lett. 92, 105501 (2004).

${ }^{4}$ F. Liu, P. Rugheimer, E. Mateeva, D. E. Savage, and M. G. Lagally, Nature (London) 416, 498 (2002).

${ }^{5}$ R. J. Hamers, Nature (London) 412, 489 (2001).

${ }^{6}$ X. F. Duan, C. M. Niu, V. Sahi, J. Chen, J. W. Parce, S. Empedocles, and J. L. Goldman, Nature (London) 425, 274 (2004).

${ }^{7}$ M. C. Mcalpine, R. S. Friedman, and C. M. Lieber, Proc. IEEE 93, 1357 (2005).

${ }^{8}$ K. Bradley, J. C. P. Gabriel, and G. Grüner, Nano Lett. 3, 1353 (2003).

${ }^{9}$ K. H. Ahn, T. Lookman, and A. R. Bishop, Nature (London) 428, 401 (2004).

${ }^{10}$ H. E. Romero, K. Bolton, A. Rosén, and P. C. Eklund, Science 307, 89 (2005).

${ }^{11}$ X. Lin, X. B. He, J. L. Lu, L. Gao, Q. Huan, D. X. Shi, and H.-J. Gao, Chin. Phys. 14, 1536 (2005).

${ }^{12}$ X. Lin, X. B. He, J. L. Lu, L. Gao, Q. Huan, Z. T. Deng, Z. H. Cheng, D. X. Shi, and H. J. Gao, Surf. Interface Anal. 38, 1096 (2006).

${ }^{13}$ F. Liu, P. J. Cao, H. R. Zhang, J. Q. Li, and H.-J. Gao, Nanotechnology 15, 949 (2004).

${ }^{14}$ H. Kind, H. Q. Yan, B. Messer, M. Law, and P. D. Yang, Adv. Mater. (Weinheim, Ger.) 14, 158 (2002).

${ }^{15}$ F. Liu, M. H. Huang, P. P. Rugheimer, D. E. Savage, and M. G. Lagally, Phys. Rev. Lett. 89, 136101 (2002).

${ }^{16}$ M. Huang, P. Rugheimer, M. G. Lagally, and F. Liu, Phys. Rev. B 72, 085450 (2005).

${ }^{17}$ U. Koch, A. Fojtik, H. Weller, and A. Henglein, Chem. Phys. Lett. 122, 507 (1985).

${ }^{18}$ S. Monticone, R. Tufeu, and A. V. Kanaev, J. Phys. Chem. B 102, 2854 (1998).

${ }^{19}$ E. M. Wong and P. C. Searson, Appl. Phys. Lett. 74, 2939 (1999).

${ }^{20}$ B. Yao, H. Shi, H. Bi, and L. Zhang, J. Phys.: Condens. Matter 12, 6265 (2000).

${ }^{21}$ K. Vanheusden, W. L. Warren, C. H. Seager, D. R. Tallant, J. A. Voigt, and B. E. Gnade, J. Appl. Phys. 79, 7983 (1996).

${ }^{22}$ Y. Li, G. S. Cheng, and L. D. Zhang, J. Mater. Res. 15, 2305 (2000).

${ }^{23}$ Q. H. Li, Q. Wan, Y. X. Liang, and T. H. Wang, Appl. Phys. Lett. 84, 4556 (2004).

${ }^{24}$ M. S. Arnold, P. Avouris, Z. W. Pan, and Z. L. Wang, J. Phys. Chem. B 107, 659 (2003). 